\title{
An Exchange of Messages Between Two Authors and a Journal
}

\section{Adolfo Quirós (Universidad Autónoma de Madrid, Spain), Chair of the EMS Ethics Committee}

\section{Background}

This note was prepared by the members of the EMS Ethics Committee. We shall describe the experience of two young European mathematicians when they submitted a manuscript as a pdf file to a mathematical journal. We shall not mention any identifying details. ${ }^{1}$ We shall refer to this mathematical journal as "the Journal". It is a journal published by a large commercial onlineonly publisher.

\section{The events}

A manuscript is submitted to the Journal. The next day, the submission is acknowledged by the Managing Editor. Fourteen days after submission, the authors are informed that their manuscript has been accepted for publication and they are asked to provide the Word or LaTeX file. They are also told how to pay the publication charge. For the purpose of this exposition, we shall assume that the charge is EUR 1000. The actual charge is slightly less.

The Managing Editor sends a reminder 18 days later.

The authors do not respond immediately. Five weeks after the submission date, and thus three weeks after acceptance, they write to the Managing Editor withdrawing their submission. They have not sent the Word or LaTeX file to the Journal.

Three days later, the Managing Editor responds that the Journal is an open-access journal and relies on publication charges to run its organisation. The authors are then offered a $20 \%$ reduction on the publication charge.

The authors respond the same day, maintaining that they have withdrawn their submission.

Two days later, they are informed that the Journal has finished the publication process and that the charge for withdrawing the submission is $50 \%$ of the publication charge. They are given the payment information.

The authors object immediately, saying that they do not understand how the journal can finish the publication process without the Word or LaTeX file. They insist that they have withdrawn their manuscript. They state that they intend to submit it to another journal.

The Managing Editor replies that the authors did not react immediately to the information that the manuscript had been accepted and then writes:

“...as DOI link have been generated you cannot submit your article in any of another Journals.”

\footnotetext{
1 The Ethics Committee has had access to the full correspondence. The quotes given are verbatim.
}

This time they are offered a $30 \%$ reduction in the publication charge.

A new person now enters the correspondence. It is someone from the management of the organisation, reminding them that they have not paid the invoice for their publication charge. This invoice is for the original amount of EUR 1000. The message is sent two weeks after the previous message from the Managing Editor.

The authors respond, stating that they have withdrawn their manuscript. The person from the management responds by offering a $20 \%$ reduction to the publication charge. He continues:

\section{"If you still wish to publish your article in our esteemed journal kindly quote the amount that you can afford, so that we can respond you positively include your valuable submission for the next issue."}

At this point, the authors decide to check the Journal. The manuscript has already been published. According to the information in the Journal, the manuscript was accepted eight days after submission and published 11 days after submission. The published version is the submitted pdf file.

They write again to the Journal pointing out that they have not transferred copyright to the Journal, but they do not receive a reply immediately.

The response that they finally receive is from a different Managing Editor. It is sent 69 days after submission. They are informed (again) that their manuscript has been accepted for publication. Two referee reports are included. The full text is (with the topic replaced by $\mathrm{X}$ ):

Comments from the Editors and Reviewers:

Reviewer \#1: I will accept this paper.

Reviewer \#2: Dear Author, Discussion of $X$ is very good.

In this mail, the publication charge is not mentioned.

\section{Lessons to be learned}

When you submit a manuscript to a journal, you should check what kind of journal it is and what conditions it imposes.

In this example, the authors did not realise that there was a publication charge since they did not check the Journal carefully. The Journal also clearly states its policy on withdrawal:

"... an author is free to withdraw an article at no charge - as long as it is withdrawn within 10 days of its initial submission." 
We note that the publication date in the Journal is 11 days after submission.

The Journal has an Editorial Board, and some prominent mathematicians are listed as members. One could wonder whether these people know this and are fully aware of the practices of this journal.

Finally, let us point out two relevant items from the Comments on the Code of Practice on the EMS homepage. We cite them here:

[PE1] Those colleagues who allow their names to be used, for example as "Editorial advisers", to assure the mathematical public of the quality of a journal, have an obligation to be well aware of, and content with, the journal's goals, policies, standards, and pricing.

We caution that the policies of journals may change with time, for example, when new owners take over, but that journals may not trouble to inform editorial advisers that their policies are changing.
[PE5] It is gross misconduct for an editor or publisher to accept for publication a submitted article without seeking to verify that the article does not plagiarize an existing article or work and is mathematically correct.

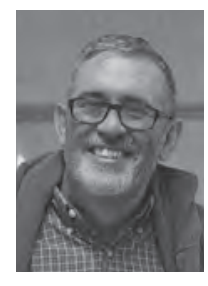

Adolfo Quirós has been a member of the EMS Ethics Committee since it was established in 2010, and serves as its Chair in 2016-2017. He obtained his Ph. D. from the University of Minnesota and is Profesor Titular at the Department of Mathematics of Universidad Autónoma de Madrid (Spain). His area of research is Arithmetic Algebraic Geometry and he is also involved in popularization activities, like the Mathematical Challenges run (on video) by the Spanish daily El País. He has been Vice-president of Real Sociedad Matemática Española and is currently editor of its members' journal, La Gaceta. 